Check for updates

Cite this: Mater. Adv., 2022, 3, 1280

Received 20th October 2021 , Accepted 10th December 2021

DOI: $10.1039 / \mathrm{d} 1 \mathrm{ma00972a}$

rsc.li/materials-advances

\title{
Photochromic behavior of diarylbenzene nanoparticles prepared by top-down and bottom-up approaches $\dagger$
}

\author{
Shota Hamatani, (D) Daichi Kitagawa, (D) * Rikuto Maegawa and Seiya Kobatake (D) * \\ The use of photochromic molecules as active elements in aggregates is very important for developing \\ advanced materials in the next generation. The nanoparticle is one of the aggregate forms and has been \\ attracting much attention due to not only the ease of preparation but also the characteristic photoswitching \\ properties. In this work, we fabricated nanoparticles composed of a photochromic diarylbenzene by both ball- \\ milling and reprecipitation methods and investigated their photochromic behaviors. The nanoparticles made by \\ the ball-milling method had crystallinity, while those made by the reprecipitation method were amorphous. \\ Both nanoparticles exhibited reversible T-type photochromic behavior, but the kinetics of the thermal back \\ reaction depended on the state of the nanoparticles. The results in this study provide a convenient and useful \\ way to prepare nanoparticles with different thermal back reactivity from one molecule.
}

\section{Introduction}

Photochromic molecules change their physicochemical properties including absorption spectra, refractive indices, dielectric constants, and oxidation-reduction potentials in response to light stimulation and have many potential applications ranging from optical data storage to bioimaging. ${ }^{1-13}$ The use of photochromic molecules can be simply divided into two types: as a switch at the single molecule level or as active elements in aggregates. Specifically, the latter is very interesting for developing advanced materials in the next generation. For instance, one of the typical forms of aggregates is crystals. Molecular structural change of the individual molecules associated with photochemical reactions in crystals leads to macroscopic changes in crystal shape. ${ }^{14-16}$ Such crystals can be used for photoactuators that do not need any electronic wires and physical contact. Another representative application form of aggregates is nanoparticles. Nanoparticles composed of photochromic molecules and fluorophores exhibit efficient photoreversible fluorescence ON/ OFF switching behavior based on Förster resonance energy transfer from a fluorophore to a photochromic molecule. ${ }^{17,18}$ These nanoparticles can be utilized for super-resolution fluorescence microscopy. Furthermore, recently, it was reported that intense nanosecond pulse laser excitation to nanoparticles composed of

Department of Applied Chemistry, Graduate School of Engineering,

Osaka City University, 3-3-138 Sugimoto, Sumiyoshi-ku, Osaka 558-8585, Japan.

E-mail: kitagawa@osaka-cu.ac.jp, kobatake@a-chem.eng.osaka-cu.ac.jp;

Fax: +8166605 2797; Tel: +81666052797

$\dagger$ Electronic supplementary information (ESI) available: Detailed experimental data (Fig. S1-S5), and the movie of the photochromic behavior for NP-ball and NP-rep (Videos S1 and S2). See DOI: 10.1039/d1ma00972a photochromic diarylethene molecules induced an amplified ringopening reaction based on the "photosynergetic response" coupled with nanoscale laser heating (photothermal) effect and photochemical reaction. ${ }^{19}$ These amplified photoswitching property is thanks to the environment in nanoparticles that molecules densely exist. Therefore, the investigation of photochromic behavior in aggregates is essential for the development of novel functional materials.

Recently, we have developed diarylbenzene (DAB) molecules as a new family of T-type photochromic molecules. ${ }^{20-22}$ DABs undergo $6 \pi$-electron photocyclization and thermal back reaction between colorless open-ring isomers and colored closedring isomers. We have revealed that the thermal back reactivity of DABs can be tuned by introducing electron-donating groups at the lateral phenyl rings or changing aryl groups. ${ }^{23}$ Moreover, with the aid of quantum chemical calculations, we have succeeded in controlling the molecular conformation at the ground state into the photoreactive antiparallel conformation, which resulted in the near photon-quantitative photocyclization in solution and fast T-type photochromism in the crystalline state. ${ }^{24,25}$ Thus, DAB molecules have great potential as a photochromic molecule. However, the photochromic behavior of nanoparticles made from DABs has been unexplored. Since the preparation of photochromic nanoparticles can extend the range of applications, we decided to fabricate nanoparticles composed of a DAB and investigate their photochromic behavior.

In this work, we report the preparation and characterization of nanoparticles composed of diarylbenzene 1a (Scheme 1) and investigated their photochromic behaviors. The top-down (ballmilling) and bottom-up (reprecipitation) approaches resulted 

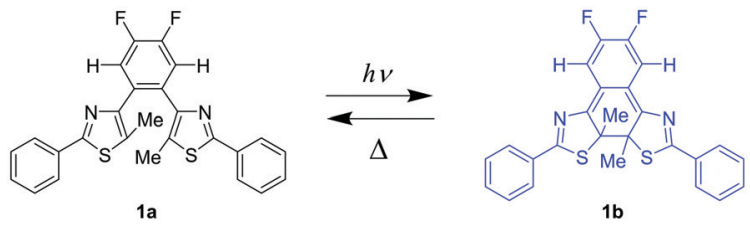

Scheme 1 Molecular structure of diarylbenzene investigated in this work.

in different nanoparticles that are crystalline and amorphous, and the rate constant of the thermal back reaction depended on the state of nanoparticles. The results in this study provide a convenient and useful way to prepare nanoparticles with different thermal back reactivity from one molecule.

\section{Results and discussion}

To make nanomaterials, there are top-down and bottom-up approaches. In this work, we adopted a ball-milling method ${ }^{26}$ and a reprecipitation method ${ }^{27}$ as the approaches (Scheme 2). First, we fabricated nanoparticles composed of 1a by a ballmilling approach (hereafter denoted as NP-ball) as detailed in experimental section and Fig. S1 (ESI $\dagger$ ). Briefly, the single crystals of 1a were crushed into small pieces in water with sodium dodecyl sulfate (SDS). Then, after centrifugation, the supernatant of the solution was collected. The solution was slight white turbid, but it remained after 1 week without precipitation. The size distribution of NP-ball just after fabrication was obtained by the dynamic light scattering (DLS) measurement, showing that the $Z$-average was $194 \mathrm{~nm}$, and the poly dispersity index (PDI) was 0.298 (Fig. 1a). Those values slightly changed to $207 \mathrm{~nm}$ and 0.259 after 1 day (Fig. 1b), meaning that NP-ball were stably dispersed in water. Thus, we succeeded in preparing nanoparticles by top-down approach. Second, we tried to make nanoparticles composed of $\mathbf{1 a}$ by a reprecipitation method (hereafter denoted as NP-rep) as detailed in the experimental section. As reported in the previous papers on fabricating nanoparticles by the reprecipitation method, ethanol was chosen as a solvent that is miscible with

(a) Top-down approach

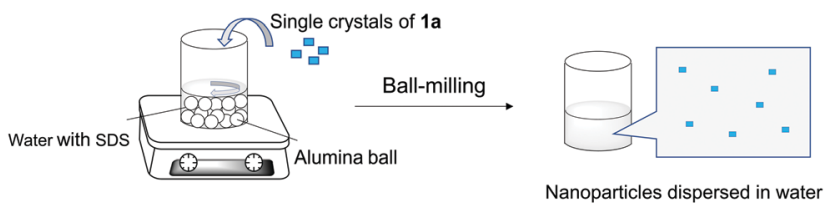

(b) Bottom-up approach

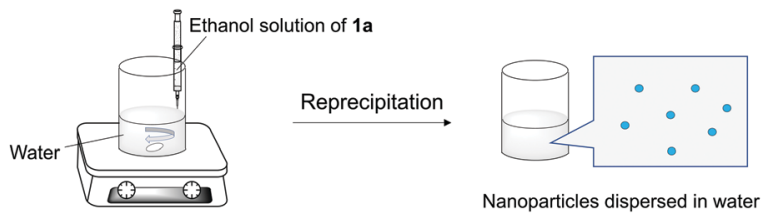

Scheme 2 Schematic illustration of (a) top-down and (b) bottom-up approaches for fabricating nanoparticles dispersed in water.
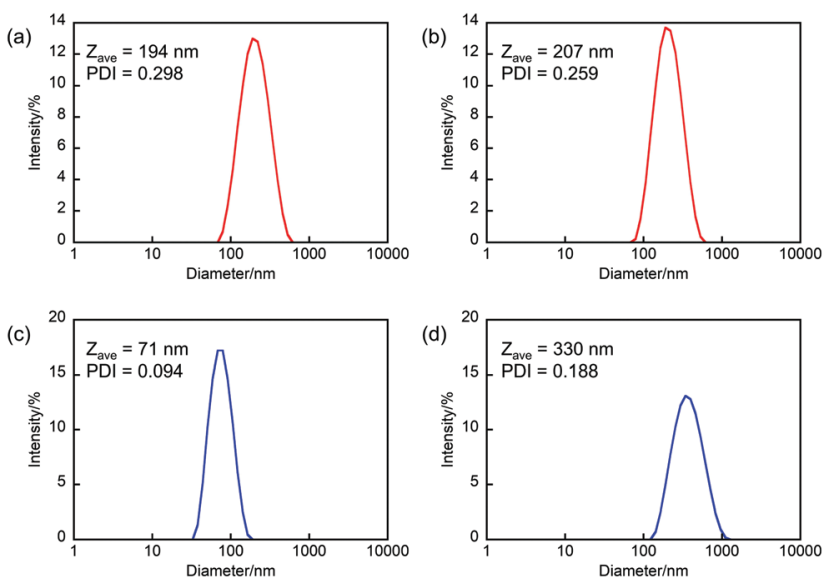

Fig. 1 DLS size distribution of ( $a$ and b) NP-ball and ( $c$ and d) NP-rep in water ( $a$ and $c$ ) just after fabrication and ( $b$ and $d$ ) after 1 day.

water and a tiny amount of the ethanol solution of 1a was injected in the vigorously stirred water by a micro-syringe. After further stirring, nanoparticles suspended in water could be obtained. The size distribution of NP-rep was also obtained by the DLS measurement, showing that the $Z$-average was $71 \mathrm{~nm}$ and the PDI was 0.094 (Fig. 1c). Those values changed to $330 \mathrm{~nm}$ and 0.188 after 1 day (Fig. 1d), but NP-rep were stably dispersed in water even after 1 week without obvious precipitation. Thus, fabricating nanoparticles by bottom-up approach was also accomplished.

To confirm that the obtained nanoparticles are amorphous or crystalline, we performed powder X-ray diffraction (PXRD) measurement (Fig. 2). In the case of NP-ball, to prevent the crystallization of excessive SDS, centrifugation and redispersion into water were performed for 3 cycles. Then, the dispersion solution of NP-ball was dropped onto a slide glass to perform PXRD measurement. Interestingly, as can be seen, PXRD on NP-ball showed intense peaks corresponding to the calculated pattern from single crystal XRD data of 1a (Fig. 2a), while PXRD

(a)

(b)

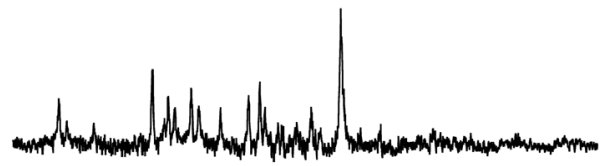

(c)
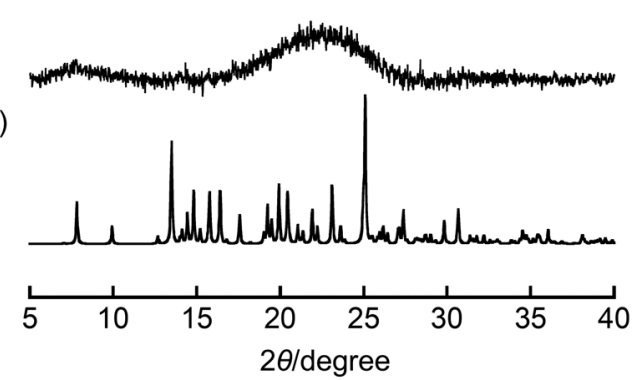

Fig. 2 PXRD pattern of (a) NP-ball, (b) NP-rep, and (c) the calculated pattern from single crystal XRD data of $1 \mathrm{a}$. 
on NP-rep did not show the obvious peaks (Fig. 2b). These results indicate that NP-ball has crystallinity, while NP-rep is amorphous. Thus, it was revealed that the different approaches for fabricating nanoparticles of DAB result in the different state nanoparticles.

Next, we explored the photochromic and the thermal back reactivities of NP-ball and NP-rep (Fig. 3 and Videos S1 and S2, ESI $\dagger$ ). Upon irradiation with $313 \mathrm{~nm}$ light to the dispersed solution of NP-ball and NP-rep, the new absorption band appeared around $680 \mathrm{~nm}$ for NP-ball and $670 \mathrm{~nm}$ for NP-rep, respectively. These spectral changes are ascribed to the photocyclization reaction from the open-ring isomer 1a to the closedring isomer 1b. After ceasing the UV irradiation, the photogenerated closed-ring isomers underwent thermal back reaction to the initial open-ring isomers, reducing the absorbance in the visible region. This spectral change could be repeated multiple times. Thus, both nanoparticles exhibited reversible T-type photochromic behavior. On the other hand, interestingly, the rate of the thermal back reaction of NP-ball and NP-rep was totally different. For NP-ball, the absorption band in the visible region quickly decreased after ceasing UV light. On the other hand, for NP-rep, the absorbance gradually decreased after stopping the UV irradiation. These results clearly indicate that the thermal back reactivity depends on the fabrication methods of nanoparticles. This is due to that the photogenerated closed-ring isomer in the crystal of the open-ring isomer is highly distorted and destabilized as reported previously. ${ }^{28}$ Therefore, the energy difference between the open- and closed-ring isomer becomes large, and the activation energy of the thermal back reaction becomes small compared with those in amorphous. As a result, the thermal back reaction in crystal is accelerated. Furthermore, in our previous work, it was
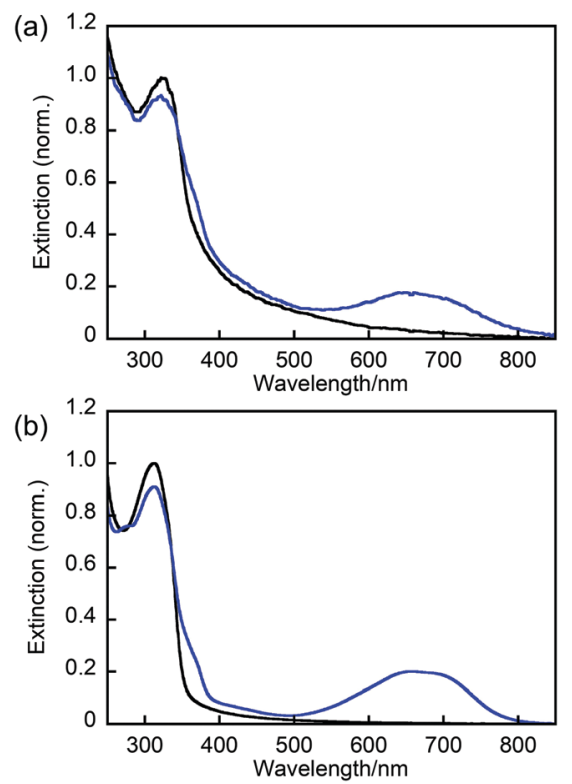

Fig. 3 Absorption spectral change of (a) NP-ball and (b) NP-rep in water: open-ring isomer (black line) and the solution under irradiation with $313 \mathrm{~nm}$ light (blue line). revealed that the photocyclization quantum yield of $\mathbf{1 a}$ in $n^{-}$ hexane is over 0.9 because of the stabilization of the photoreactive anti-parallel conformation by the intramolecular $\mathrm{CH}-\mathrm{N}$ hydrogen bonding interactions. ${ }^{24}$ Therefore, it can be concluded that the difference in the coloration of nanoparticles in the ESI $\dagger$ Videos is ascribed to the difference in the thermal back reaction rate. In other words, the rate of the thermal back reaction in the crystalline nanoparticles is faster than that in the amorphous nanoparticles, resulting in the lower coloration.

To quantitatively evaluate the thermal back reactivity, we measured the change in the absorption for NP-ball and NP-rep as a function of time at $298 \mathrm{~K}$ (Fig. 4). Note that freshly prepared samples were used for the following experiments, but it was confirmed that similar results were obtained with the samples stored for a few days. As can be seen, the absorbance for NP-ball decreased to 0.2 at $25 \mathrm{~s}$, while that for NP-rep decreased to 0.2 at $1200 \mathrm{~s}$. There is a large difference in the thermal back reactivity between NP-ball and NP-rep. Furthermore, interestingly, although the kinetics for the thermal back reaction of $\mathbf{1 b}$ in solution and in single crystal followed monoexponential as reported previously, ${ }^{24}$ that for NP-ball and NP-rep did not obey monoexponential. It is generally known that the reaction rate of photochromism is influenced by the environment around the photochrome. ${ }^{29,30}$ Therefore, these results imply that some populations having different thermal back reaction rates exist in NP-ball and NP-rep.

To mathematically analyze the absorption decay curve for NP-ball and NP-rep, we performed the fitting using the following biexponential equation that is well used for the analysis of
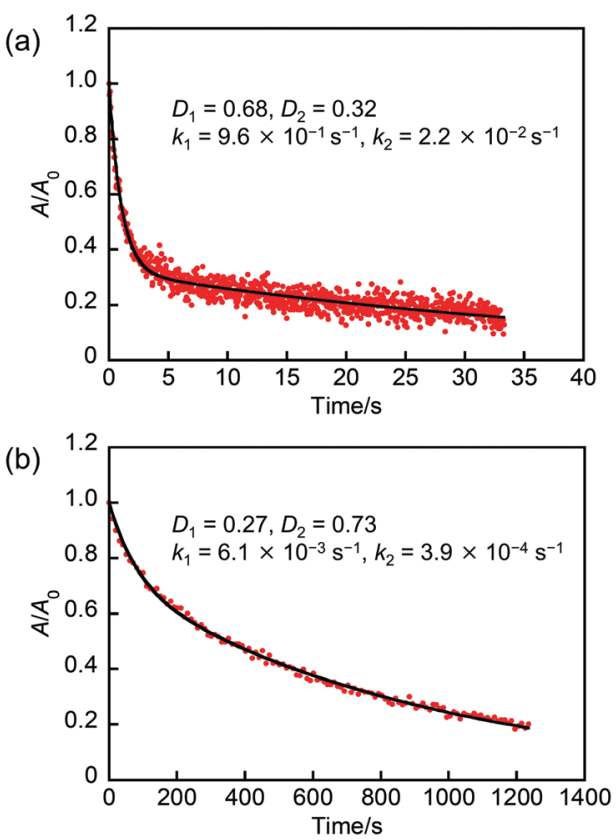

Fig. 4 Absorption decay curves at $\lambda_{\max }$ for (a) NP-ball and (b) NP-rep in water at $298 \mathrm{~K}$. Red plots indicate experimental values. Black solid lines indicate the theoretical curves of the fitting using the biexponential equation. 
the thermal back reaction of photochromic compounds in polymer matrices; ${ }^{31-33}$

$$
D(t)=D_{1} e^{-k_{1} t}+D_{2} e^{-k_{2} t}
$$

where $D(t)$ is the optical density at the $\lambda_{\max }, D_{1}$ and $D_{2}$ are contributions to the initial optical density $D_{0}, k_{1}$ and $k_{2}$ are the rate constants of the two components. The absorption decay curves of NP-ball and NP-rep were well described by the biexponential equation and the rate constants obtained by the fitting are summarized in Table 1 . In the case of NP-ball, the obtained values were $k_{1}=9.6 \times 10^{-1} \mathrm{~s}^{-1}\left(D_{1}=0.68\right)$ and $k_{2}=2.2 \times 10^{-2} \mathrm{~s}^{-1}\left(D_{2}=0.32\right)$. This result indicates that $68 \%$ of molecules in NP-ball exhibited the faster thermal back reaction and the residual $32 \%$ molecules exhibited the slower one. Similarly, the values obtained for NP-rep were $k_{1}=6.1 \times$ $10^{-3} \mathrm{~s}^{-1}\left(D_{1}=0.27\right)$ and $k_{2}=3.9 \times 10^{-4} \mathrm{~s}^{-1}\left(D_{2}=0.73\right)$, meaning that $27 \%$ of molecules in NP-rep exhibited the faster thermal back reaction and the residual $73 \%$ molecules exhibited the slower one. Thus, the existence of at least two populations with different thermal back reaction rate was confirmed in both NP-ball and NP-rep.

To interpret the biexponential behavior in detail, we compared the values of the rate constant in NP-ball and NP-rep with that in $n$-hexane and in single crystal, which are also summarized in Table 1 . First, in the case of NP-ball, the $k_{1}$ value is close to the rate constant in single crystal. This indicates that there is an environment similar to single crystal in NP-ball, which is consistent with the result of PXRD measurement described above. On the other hand, the $k_{2}$ value in NP-ball is smaller than that in single crystal, but larger than that in $n$-hexane. In addition, it is also different from the $k_{1}$ and $k_{2}$ values for NP-rep that is amorphous. These results suggest that the slower component in NP-ball is a state with low crystallinity. This would be due to the fabrication procedure of NP-ball. One is that the ball-milling brought about the low crystallinity part such as the surface of nanoparticles. Another possibility is the existence of SDS in water. It is previously reported that SDS affect the crystallinity of molecular crystals. ${ }^{34}$ The loose packing in the low crystallinity environment would lead to a less distorted closed-ring isomer compared with that in the high crystallinity environment, resulting in a smaller rate constant of the thermal back reaction. Actually, the kinetics of the thermal back reaction in powder crystals prepared by cracking a large single crystal (Fig. S2, ESI $\dagger$ ) followed biexponential as shown in

Table 1 The rate constants of the thermal back reaction for the photogenerated closed-ring isomer in various states at $298 \mathrm{~K}$

\begin{tabular}{llllll}
\hline & $k / \mathrm{s}^{-1}$ & $D_{1}$ & $D_{2}$ & $k_{1} / \mathrm{s}^{-1}$ & $k_{2} / \mathrm{s}^{-1}$ \\
\hline In $n$-hexane & $2.5 \times 10^{-3}$ & - & - & - & - \\
In single crystal & 1.2 & - & - & - & - \\
In NP-ball & - & 0.68 & 0.32 & $9.6 \times 10^{-1}$ & $2.2 \times 10^{-2}$ \\
In NP-rep & - & 0.27 & 0.73 & $6.1 \times 10^{-3}$ & $3.9 \times 10^{-4}$ \\
In powder crystals & - & 0.85 & 0.15 & 1.0 & $1.8 \times 10^{-2}$ \\
In PMMA & - & 0.39 & 0.61 & $5.8 \times 10^{-3}$ & $9.0 \times 10^{-4}$ \\
In PIBMA & - & 0.32 & 0.68 & $7.8 \times 10^{-3}$ & $1.2 \times 10^{-3}$ \\
In PBA & $2.7 \times 10^{-3}$ & - & - & - & -
\end{tabular}
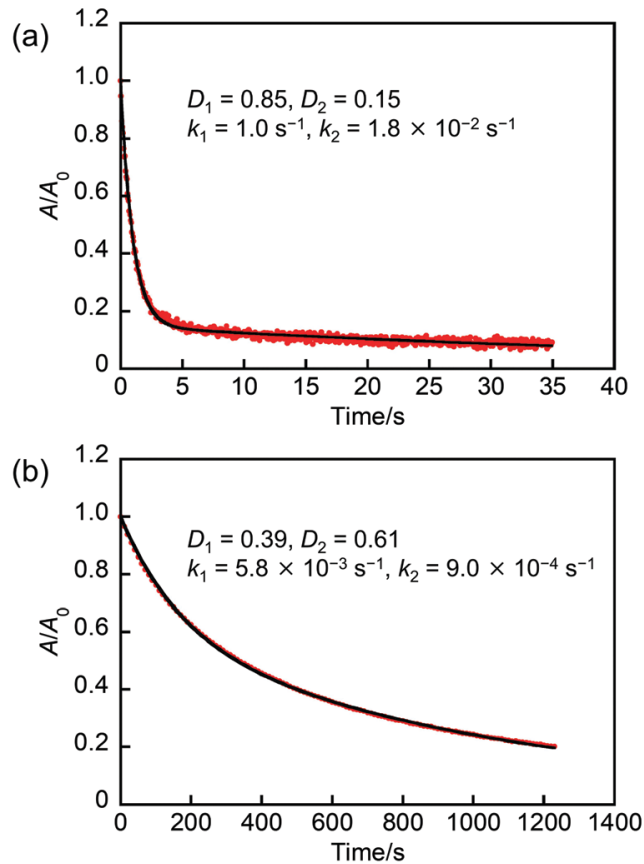

Fig. 5 Absorption decay curves at $\lambda_{\max }$ for (a) powder crystals and (b) PMMA film at $298 \mathrm{~K}$. Red plots indicate experimental values. Black solid lines indicate the theoretical curves of the fitting using the biexponential equation.

Fig. 5a. The $k_{1}$ and $k_{2}$ values in powder crystals were $1.0 \mathrm{~s}^{-1}$ and $1.8 \times 10^{-2} \mathrm{~s}^{-1}$, which is very similar to that in NP-ball. This result supports the discussion described above.

Next, as for NP-rep, the $k_{1}$ value is nearly close to the rate constant in $n$-hexane. On the other hand, the $k_{2}$ value in NP-rep is very small compared with others. To explain this, we hypothesize that the inhomogeneity of the free-volume around the molecules within NP-rep would result in the formation of two different environments for the photogenerated closed-ring isomers. One is where the molecules can change their geometrical structure easily and quickly, leading to the rate constant of the thermal back reaction similar to that in solution. The other is where the molecules can only slowly change their geometrical structure, resulting in a rate constant very slower than that in solution. To confirm the validity of our hypothesis, the thermal back reactivity in a polymer was investigated. It is reported that there is an inhomogeneity of the free-volume in a polymer film. ${ }^{33,35}$ Therefore, the investigation of the thermal back reactivity in a polymer film would provide precious information. Here, we selected three types of polymers with various glass transition temperatures $\left(T_{\mathrm{g}}\right)$, poly(methyl methacrylate) (PMMA: $T_{\mathrm{g}}=c a .378 \mathrm{~K}$ ), poly(isobutyl methacrylate) (PIBMA: $T_{\mathrm{g}}=c a .326 \mathrm{~K}$ ), and poly(butyl acrylate) (PBA: $T_{\mathrm{g}}=c a$. $224 \mathrm{~K})$. The preparation of polymer films containing 1a was described in the experimental section in detail. Fig. 5b shows the absorption decay curves of $\mathbf{1 b}$ in PMMA film after ceasing UV irradiation at $298 \mathrm{~K}$. The thermal back reaction kinetics in PMMA film followed biexponential and the $k_{1}$ and $k_{2}$ values in PMMA film were $5.8 \times 10^{-3} \mathrm{~s}^{-1}$ and $9.0 \times 10^{-4} \mathrm{~s}^{-1}$, which is 
very similar to that in NP-rep. The similar results were also obtained in PIBMA film as shown in Fig. S3 (ESI $\dagger$ ), although the $k_{1}$ and $k_{2}$ values slightly changed to $7.8 \times 10^{-3} \mathrm{~s}^{-1}$ and $1.2 \times 10^{-3} \mathrm{~s}^{-1}$, respectively. On the other hand, the thermal back reaction kinetics in PBA film followed monoexponential as shown in Fig. S4 (ESI $\dagger$ ), and the $k$ value in PBA film was $2.7 \times 10^{-3} \mathrm{~s}^{-1}$, which is very similar to that in $n$-hexane. These results suggest that the polymers with $T_{\mathrm{g}}$ higher than the measurement temperature $(298 \mathrm{~K})$ bring about the inhomogeneity of the free-volume around the molecules, which leads to the biexponential character for the thermal back reaction kinetics. In contrast, the polymer with $T_{\mathrm{g}}$ lower than $298 \mathrm{~K}$ results in the monoexponential kinetics due to the absence of the inhomogeneity. The $T_{\mathrm{g}}$ of the amorphous solid of 1a was determined to be $c a .311 \mathrm{~K}$ by a differential scanning calorimetry (DSC) measurement as shown in Fig. S5 (ESI $\dagger$ ), which is higher than the measurement temperature (298 K). Therefore, the thermal back reaction kinetics in NP-rep followed biexponential. Thus, the kinetics of the thermal back reaction in NP-rep could be successfully interpreted from the viewpoint of the inhomogeneity of the free-volume around the molecules within nanoparticles.

There are two notable aspects in this work. One is that the state of nanoparticles depends on the fabricating procedure of nanoparticles. Usually, to fabricate crystalline nanoparticles by top-down approach, compounds with high crystallinity are suitable. ${ }^{27}$ Therefore, when compounds with high crystallinity are used, the reprecipitation method also gives crystalline nanoparticles. However, in the case of DAB 1a, we fortunately found that the state of nanoparticles can be controlled by changing the fabrication method. The other is that the thermal back reactivity of DAB drastically changes depending on the state of nanoparticles. In general, to obtain different thermal back reactivity, it is necessary to design and synthesize different compounds. Our results provide a convenient and useful way to prepare nanoparticles with different thermal back reactivity from one molecule. Furthermore, the ability to exhibit different thermal back reactivity depending on the state of nanoparticles might be utilized for the detection of the change in microenvironments. We are now investigating the potential applications of the DAB nanoparticles.

\section{Experimental}

\section{General}

Solvents used were of spectroscopic grade or purified by distillation before use. The centrifugation was performed by a TOMY Suprema21 or an Eppendorf MiniSpin. PXRD profiles were recorded on a Rigaku MiniFlex 600 diffractometer using $\mathrm{Cu} \mathrm{K}_{\alpha}$ radiation $(\lambda=1.54184 \AA)$. The nanoparticle size was determined by the DLS (Sysmex Zetasizer Nano ZS) analysis. DSC was performed using a Hitachi DSC7000X instrument. UVVis absorption spectra were measured using a JASCO V-560 absorption spectrometer or an Ocean Optics FLAME-S multichannel analyzer. Temperature control during the absorption spectroscopic measurement was performed with a UNISOKU CoolSpeK UV/CD or an Ocean Optics CUV-QPOD. Photoirradiation was carried out using a $300 \mathrm{~W}$ xenon lamp (Asahi Spectra MAX-301) as a light source. Monochromatic light (313 nm light) was obtained by passing light through a band-pass filter. The image of powder crystals was taken by a Keyence VHX-500 Digital Microscope.

\section{Materials}

Chemicals used for synthesis were commercially available and used without further purification. 1a was synthesized by the method reported previously. ${ }^{24}$

\section{Preparation of NP-ball}

$5 \mathrm{~mL}$ of a sodium dodecyl sulfate (SDS) aqueous solution (1.4 mM) was added into a $30 \mathrm{~mL}$ glass vial. A stir bar, 30 alumina balls $(\phi=5 \mathrm{~mm})$ and single crystals $1 \mathrm{a}(11 \mathrm{mg})$ were added into the vial. The mixture was vigorously stirred overnight and the water portion in which the DAB was dispersed was centrifuged to precipitate large particles $(6000 \mathrm{rpm}$ for $5 \mathrm{~min}$ ). The supernatant was collected and used as NP-ball dispersion solution.

\section{Preparation of NP-rep}

$1.2 \mathrm{mg}$ of $1 \mathrm{a}$ was dissolved into $1 \mathrm{~mL}$ of ethanol with sonication for $10 \mathrm{~min}$. $200 \mu \mathrm{L}$ of the ethanol solution of $1 \mathrm{a}\left(2.6 \times 10^{-6} \mathrm{M}\right)$ was injected into vigorously stirred water $(10 \mathrm{~mL})$ by a microsyringe, resulting in NP-rep dispersion solution.

\section{Preparation of polymer films containing 1a}

Each Polymer was dissolved in toluene to prepare a $12 \mathrm{wt} \%$ toluene solution containing polymer. 1a was added to the solution to prepare a toluene solution containing $1 \mathbf{a}$ and polymer $(1: 24 \mathrm{w} / \mathrm{w})$. The solution was casted on a quartz glass and dried in air. Then, the film was annealed at $160{ }^{\circ} \mathrm{C}$ for $1 \mathrm{~h}$.

\section{Conclusions}

In this study, we prepared nanoparticles composed of photochromic diarylbenzene by top-down and bottom-up approaches: ball-milling and reprecipitation methods. The nanoparticles made by the ball-milling method provided crystalline nanoparticles, while the reprecipitation method resulted in amorphous nanoparticles. Both types of nanoparticles exhibited reversible T-type photochromism, but the rate constants of the thermal back reaction were different from each other. The kinetics of the thermal back reaction in nanoparticles were successfully interpreted by the difference in the environment around molecules. The results in this study provide a convenient and useful way to prepare nanoparticles with different thermal back reactivity from one molecule. 


\section{Author contributions}

Shota Hamatani: investigation, formal analysis, visualization, writing - original draft; Daichi Kitagawa: conceptualization, methodology, formal analysis, project administration, resources, supervision, writing - original draft, writing - review \& editing; Rikuto Maegawa: investigation, formal analysis, visualization; Seiya Kobatake: resources, project administration, supervision, writing - review \& editing.

\section{Conflicts of interest}

There are no conflicts to declare.

\section{Acknowledgements}

We thank Dr Hikaru Sotome (Osaka University) for useful scientific discussions. This work was partly supported by JSPS KAKENHI Grant Numbers 21K14603 for D. K. and 21H02016 for S. K.

\section{Notes and references}

1 H. Dürr and H. Bouas-Laurent, Photochromism: Molecules and Systems, Elsevier, Amsterdam, 2003.

2 H. Bouas-Laurent and H. Durr, Pure Appl. Chem., 2001, 73, 639-665.

3 M. Irie, T. Fukaminato, K. Matsuda and S. Kobatake, Chem. Rev., 2014, 114, 12174-12277.

4 T. Fukaminato, T. Umemoto, Y. Iwata, S. Yokojima, M. Yoneyama, S. Nakamura and M. Irie, J. Am. Chem. Soc., 2007, 129, 5932-5938.

5 T. Fukaminato, T. Sasaki, T. Kawai, N. Tamai and M. Irie, J. Am. Chem. Soc., 2004, 126, 14843-14849.

6 K. Klaue, W. Han, P. Liesfeld, F. Berger, Y. Garmshausen and S. Hecht, J. Am. Chem. Soc., 2020, 142, 11857-11864.

7 K. Klaue, Y. Garmshausen and S. Hecht, Angew. Chem., Int. Ed., 2018, 57, 1414-1417.

8 A. Kometani, Y. Inagaki, K. Mutoh and J. Abe, J. Am. Chem. Soc., 2020, 142, 7995-8005.

9 A. Tokunaga, L. M. Uriarte, K. Mutoh, E. Fron, J. Hofkens, M. Sliwa and J. Abe, J. Am. Chem. Soc., 2019, 141, 17744-17753.

10 K. Mutoh, N. Miyashita, K. Arai and J. Abe, J. Am. Chem. Soc., 2019, 141, 5650-5654.

11 K. Uno, M. L. Bossi, M. Irie, V. N. Belov and S. W. Hell, J. Am. Chem. Soc., 2019, 141, 16471-16478.

12 D. Kim, K. Jeong, J. E. Kwon, H. Park, S. Lee, S. Kim and S. Y. Park, Nat. Commun., 2019, 10, 3089.

13 X. J. Piao, Y. Zou, J. C. Wu, C. Y. Li and T. Yi, Org. Lett., 2009, 11, 3818-3821.
14 S. Kobatake, S. Takami, H. Muto, T. Ishikawa and M. Irie, Nature, 2007, 446, 778-781.

15 R. O. Al-Kaysi, A. M. Mueller and C. J. Bardeen, J. Am. Chem. Soc., 2006, 128, 15938-15939.

16 D. Kitagawa, H. Tsujioka, F. Tong, X. Dong, C. J. Bardeen and S. Kobatake, J. Am. Chem. Soc., 2018, 140, 4208-4212.

17 S. Ishida, T. Fukaminato, D. Kitagawa, S. Kobatake, S. Kim, T. Ogata and S. Kurihara, Chem. Commun., 2017, 53, 8268-8271.

18 J. Su, T. Fukaminato, J.-P. Placial, T. Onodera, R. Suzuki, H. Oikawa, A. Brosseau, F. Brisset, R. Pansu, K. Nakatani and R. Métivier, Angew. Chem., Int. Ed., 2016, 55, 3662-3666.

19 Y. Ishibashi, S. Nakai, K. Masuda, D. Kitagawa, S. Kobatake and T. Asahi, Chem. Commun., 2020, 56, 7088-7091.

20 D. Kitagawa, T. Nakahama, Y. Nakai and S. Kobatake, J. Mater. Chem. C, 2019, 7, 2865-2870.

21 S. Hamatani, D. Kitagawa, T. Nakahama and S. Kobatake, Tetrahedron Lett., 2020, 61, 151968.

22 R. Maegawa, D. Kitagawa, S. Hamatani and S. Kobatake, New J. Chem., 2021, 45, 18969-18975.

23 T. Nakahama, D. Kitagawa and S. Kobatake, J. Phys. Chem. C, 2019, 123, 31212-31218.

24 S. Hamatani, D. Kitagawa and S. Kobatake, J. Phys. Chem. C, 2021, 125, 4588-4594.

25 D. Kitagawa, N. Takahashi, T. Nakahama and S. Kobatake, Photochem. Photobiol. Sci., 2020, 19, 644-653.

26 K. Nishimura, H. Kouno, K. Tateishi, T. Uesaka, K. Ideta, N. Kimizuka and N. Yanai, Phys. Chem. Chem. Phys., 2019, 21, 16408-16412.

27 H. Kasai, H. S. Nalwa, H. Oikawa, S. Okada, H. Matsuda, N. Minami, A. Kakuta, K. Ono, A. Mukoh and H. Nakanishi, Jpn. J. Appl. Phys., 1992, 31, L1132.

28 S. Kobatake, K. Shibata, K. Uchida and M. Irie, J. Am. Chem. Soc., 2000, 122, 12135-12141.

29 T. Asano, J. Am. Chem. Soc., 1980, 102, 1205-1206.

30 T. Asano, T. Yano and T. Okada, J. Am. Chem. Soc., 1982, 104, 4900-4904.

31 R. A. Evans, T. L. Hanley, M. A. Skidmore, T. P. Davis, G. K. Such, L. H. Yee, G. E. Ball and D. A. Lewis, Nat. Mater., 2005, 4, 249-253.

32 W. Sriprom, M. Néel, C. D. Gabbutt, B. M. Heron and S. Perrier, J. Mater. Chem., 2007, 17, 1885-1893.

33 G. Such, R. A. Evans, L. H. Yee and T. P. Davis, J. Macromol. Sci., Polym. Rev., 2003, 43, 547-579.

34 F. Tong, M. Liu, R. O. Al-Kaysi and C. J. Bardeen, Langmuir, 2018, 34, 1627-1634.

35 S. Ito, Y. Taga, K. Hiratsuka, S. Takei, D. Kitagawa, S. Kobatake and H. Miyasaka, Chem. Commun., 2015, 51, 13756-13759. 